\title{
MODELLING THE ORAL GLUCOSE TOLERANCE TEST IN NORMAL AND IMPAIRED GLUCOSE TOLERANT STATES: \\ A POPULATION APPROACH
}

Michael J. Theodorakis ${ }^{1,2^{*}}$, Niki Katsiki ${ }^{3}$, Kalliopi Arampatzi ${ }^{2}$, George P. Chrousos ${ }^{2}$

1 Diabetes Trial Unit, University of Oxford, Oxford UK

2 Endocrinology, Metabolism and Diabetes Unit, Evgenideion Hospital, University of Athens, Athens Greece

${ }^{3}$ Diabetes Section, Papageorgiou Hospital, Thessaloniki Greece

*Corresponding author:

Michael J. Theodorakis, FRCP

University of Oxford Diabetes Trials Unit,

Oxford Centre for Diabetes Endocrinology and Metabolism

Churchill Hospital, Old Road, Headington, Oxford OX3 7LJ, U.K.

michael.theodorakis@dtu.ox.ac.uk

Tel: ++44 1865857230

Fax: ++441865 857241

All authors declare no conflict of interest

Keywords: population, nonlinear, mixed-effects, model, OGTT

Abstract: 294 words

Main Text: 3498 words (Supplement: 1016 words)

Four Figures; two Tables 


\section{ABSTRACT}

Objective The conventional approach to analyzing data from oral glucose tolerance testing (OGTT) requires model identification in each individual separately (standard two stage, STS), ignoring knowledge about the population as a whole. In practice, however, the OGTT is sparsely sampled and individual estimates are often not resolvable from available data. This weakness is often encountered in large scale trials or epidemiological studies, leading to either multiple imputations or simply much less data available for analysis.

Methods We have applied a population approach, nonlinear mixed effects modeling, to plasma glucose, insulin and C-peptide data obtained from a 120 minute OGTT undertaken by 106 subjects with varying glucose tolerance. This method provides estimates of population means, variances and co-variances of model parameters and empirical Bayes estimates of individual parameter values, as well as measures of intra-individual (within-subject) and inter-individual (between-subject) variability. The recently developed oral glucose minimal model was used to evaluate insulin sensitivity, and a combined model approach was used to assess $\beta$-cell secretion. Results Applying these models allowed for the reconstruction of insulin secretion and glucose absorption profiles and gave population indexes of insulin sensitivity $\left(S_{\text {I }}\right.$ $\left.=6.51 \pm 1.20 \times 10^{-4} \mathrm{~min}^{-1} \cdot \mu \mathrm{U}^{-1} \cdot \mathrm{ml}\right)$, fractional hepatic extraction of insulin $(\mathrm{F}=0.522 \pm 0.291)$ and fractional insulin clearance $\left(k_{l}=0.258 \pm 0.151 \mathrm{~min}^{-1}\right)$. Whereas the traditional approach to parameter estimation failed to recover estimates in more than one third of the population, the population approach provided individual estimates in all subjects. Examination of the empirical Bayes estimates showed that individual parameter estimates were able to differentiate well between individuals at glucose tolerant states ranging from euglycemia to overt type 2 diabetes.

Conclusions Our findings suggest population analysis is a powerful tool to obtain accurate assessments of indexes of insulin sensitivity and $\beta$-cell function from the OGTT, especially in epidemiological studies with large numbers of sparsely sampled subjects. 


\section{INTRODUCTION}

The two physiological factors whose balance is primarily responsible for maintaining normal glucose homeostasis are insulin secretion by the islet $\beta$-cells of the endocrine pancreas and insulin action. Gradual development of impaired glucose tolerance and eventually $\beta$-cell failure and type 2 diabetes, results from insulin resistance and/or $\beta$-cell dysfunction progressing over long periods of time. The most widely used tests to assess these two colluding factors in clinical practice are the clamp technique (1) and the intravenous glucose tolerance test (IVGTT) $(2,3)$. These procedures, while reliable, are in a way "non-physiological" and require frequent blood sampling and infusions of glucose and/or insulin, rendering them essentially impractical for large epidemiological or longitudinal studies. As a result, recent efforts have focused on development of indirect means of assessing insulin resistance and insulin secretion from an easily administered test reflecting everyday glucose metabolism, such as the meal tolerance test (MTT) or the oral glucose tolerance test (OGTT) $(4,5)$.

Unfortunately, some of the same qualities making the OGTT attractive for large-scale epidemiological trials (easily performed, reduced sampling) can also adversely impact parameter estimation methods (sparse data). In many cases, individual parameter estimates may not be estimable (7), or the precisions of the parameter estimates may be unsatisfactory, especially under fixed sampling intervals encompassed by the OGTT protocol. Therefore, population analysis methods can be a substantial improvement over the current parameter estimation techniques and be of particular importance when analyzing data from large clinical studies.

In general, population analysis is based upon exploiting the fact that subjects belong to a population of individuals who share certain quantitative traits. Two methods recently applied to IVGTT data are the Bayesian hierarchical approach (6) and the iterative twostage method (7). Another class of population analysis techniques is mixed effects modeling, which allows for the simultaneous estimation of population means, variances, and co-variances. Although this technique has gained recognition in the field of pharmacokinetics/pharmacodynamics $(8,9)$, it has rarely been used to estimate insulin sensitivity. One notable exception is its application (10), to a rather small number of 
healthy subjects undergoing an IVGTT, resulting in smaller parameter standard errors than a standard two-stage (STS) approach.

The aim of the present study was therefore to apply a population approach, mixed effects modeling, to existing models developed for the OGTT to effectively distinguish between varying degrees of glucose intolerance. In this manner, we sought to establish a robust tool to address a major unmet need in identifying discernible patterns among individuals at varying glucose tolerant states.

\section{MATERIALS AND METHODS}

\section{Study Population}

Our study population consists of 106 individuals whose responses span the entire spectrum of glucose tolerance states. We recruited volunteer participants from our Unit Registry to undergo a modified OGTT that was carried out after they had given written informed consent. Such tests have been completed for all participants. In accordance with the American Diabetes Association diabetes classification criteria (11), of those subjects studied, 41 had normal fasting $(<100 \mathrm{mg} / \mathrm{dl})$ and normal $2 \mathrm{~h}$ plasma glucose (<140 mg/dl; Group 1); 19 had normal fasting but impaired $2 \mathrm{~h}$ glucose tolerance (140$199 \mathrm{mg} / \mathrm{dl}$; Group 2); 20 had impaired fasting (100-125 mg/dl) and impaired $2 \mathrm{~h}$ glucose (140-199 mg/dl; Group 3); 9 had normal fasting but diabetic $2 \mathrm{~h}$ glucose levels ( $\geq 200$ $\mathrm{mg} / \mathrm{dl}$; Group 4); and 17 had newly diagnosed type 2 diabetes (by both fasting plasma glucose $\geq 126$ and $2 \mathrm{~h}$ plasma glucose $\geq 200 \mathrm{mg} / \mathrm{dl}$; group 5). Subjects were not taking oral hypoglycemic agents or insulin. Any subject on other medications was allowed to take it upon completion of the OGTT. The study was approved by the Ethics Review Board. All volunteers were advised on the nature and effects of the clinical study and they all provided written informed consent, in accordance with the Helsinki II declaration.

\section{Modified Oral Glucose Tolerance Test}

Following an overnight fast, participants drank 75 grams of glucose and blood samples were collected at baseline (time "0") and then at 5, 10, 15, 20, 40, 60, 80, 100 and 120 minutes after oral glucose, for measurements of plasma glucose, insulin, C-peptide, and glucagon levels. We used the insulinogenic index (12), calculated as the ratio of the 
increment in the plasma insulin level to that in the plasma glucose level during the first 20 minutes after ingestion of glucose, to assess early phase insulin secretion: lower indices indicate worsening insulin secretion. We also determined insulin resistance by the homeostatic model assessment (HOMA-IR) (13), calculated as the product of fasting insulin (in $\mu \mathrm{U} / \mathrm{ml}$ ) and fasting glucose (in mmol/l) divided by 22.5 . Lower ratios indicate better insulin sensitivity. The bodyweight and height of subjects were measured manually by a medical scale (SECA Corp.) and BMI $\left(\mathrm{kg} / \mathrm{m}^{2}\right)$ was calculated as bodyweight in kilograms divided by the square of the height in meters.

\section{Plasma Hormone and Biochemical Assays}

We performed plasma hormone and biochemical assays as we described previously (14). We assayed plasma samples for insulin and C-peptide by ELISA (ALPCO Diagnostics) with a detection limit of $1 \mu \mathrm{U} / \mathrm{ml}$ and $20 \mathrm{pmol}$, respectively. Cross-reactivity of the insulin antibody for C-peptide and vice versa, was less than $0.1 \%$. We measured plasma glucagon by radioimmunoassay (Linco Research), with a detection limit of $2 \mathrm{pg}$ (100 $\mu \mathrm{l}$ plasma). The intraassay coefficients of variation for insulin, C-peptide, and glucagon were $3.6,3.6$, and $4.8 \%$, respectively, and the interassay coefficients of variation were $2.5,3.3$, and $12 \%$, respectively. We measured plasma glucose levels with a glucose analyzer (Beckman Instruments) and glycosylated hemoglobin with an automated DiaSTAT analyzer (Bio-Rad Laboratories). Plasma lipid levels were determined by the Clinical Core Laboratory Unit (Evgenideion Hospital) using an AutoAnalyzer (Beckman Instruments).

\section{Univariate Statistical Analysis}

All data from the study participants were analyzed using SAS 8.2 software (SAS Institute). All values are expressed as means \pm SE. Standard methods were used to compute means and SE. All data were normally distributed (Kolmogorov and Smirnov test). One-way ANOVA and Bonferroni's multiple comparison post-hoc test were used to compare mean values of all estimated parameters among the five groups. $P$ values below 0.05 were regarded as indicating statistical significance. Population means and variances of model parameters are reported, as well as a description of between- 
subject, or inter-individual variability. Individual model parameter predictions are analyzed to determine their ability to differentiate adequately between individuals with euglycemia and varying glucose intolerance status.

Modeling approach (please see Supplement for detailed description)

\section{A. Oral Glucose Minimal Model}

The established oral glucose minimal model applied to a meal test (5) has been used to assess insulin sensitivity during the OGTT. The model assumes glucose disappearance is enhanced by elevated glucose concentration independent of insulin, referred to as glucose effectiveness, and the effect of glucose dependent on insulin acting from a compartment remote from plasma. Remote insulin is increased by plasma levels of insulin and decreased through a first-order process.

\section{B. Combined Model of Insulin and C-peptide Secretion}

A combined model of insulin and C-peptide kinetics, linked by a common equimolar prehepatic secretion rate, was used to describe $\beta$-cell function. $(15,16)$. C-peptide is assumed to reside in a central compartment and distributed to peripheral tissues. The central compartment is also referred to as the accessible compartment, since it is from this compartment that biological measurements are made.

\section{Population Model}

Parameter means, variances, and covariances were estimated using a nonlinear mixed effects model specifying two levels of variability: intra-individual variability, or the random variation among measurements within an individual, and inter-individual variability reflecting variation between different individuals.

\section{Model Analysis}

Individual profiles were initially fit using the commercially available software SAAM II (19) in order to test the structural model and obtain reasonable initial parameter estimates. All population models were run with the first-order $(F O)$ estimation method in NONMEM (20), using the POSTHOC option in order to obtain individual empirical 
Bayesian post hoc model parameter estimates. This method minimizes twice the negative marginal likelihood using a first-order linearization of the hierarchical nonlinear model (18). The three models for glucose, insulin and C-peptide were fit simultaneously. Between-individual variation was modeled with an exponential (lognormal) term for model parameters $p_{2}, \mathrm{~S}_{\mathrm{l}}, k_{/}$and $\mathrm{F}$ in order to restrain these parameter values to be positive. An additive error structure (normal) was used to model between-individual variation for rate parameters $k_{i}$ 's and $j$ 's. Due to the extensive computational time required for each NONMEM run (days), we only considered a proportional error model for the residual unknown variability, as suggested by individual model fits in SAAM II. The adequacy of the model to fit the data was determined by examining plots of predicted versus observed concentrations and weighted residuals versus time.

\section{RESULTS}

\section{Subject Characteristics}

All subjects in the study had no statistical differences in their age and body mass index. Characteristics of our study population are shown in Table 1. Subjects with type 2 diabetes had significantly higher HOMA-IR, glycosylated hemoglobin, and triglyceride levels, coupled with lower HDL cholesterol levels, compared to subjects with normal glucose tolerance (Table 1). They also had increased fasting plasma insulin levels, and a significantly reduced insulinogenic index from 0 to 20 minutes after oral glucose, compared to subjects with normal glucose tolerance (Table 1, Fig. 1). As demonstrated previously (24), fasting glucagon levels were significantly higher in subjects with type 2 diabetes (Table 1). All subjects had similar fasting plasma biochemical values and were without medical histories of gastroparesis, kidney disease or microvascular disease.

\section{Parameter Estimates}

Plots of mean concentrations of glucose, insulin and C-peptide for subsets of the population with differing levels of fasting glucose and glucose tolerance are given in Figure 1. When individual profiles were fit separately using the SAAM II software, parameter estimates were unobtainable or poorly estimated in over one-third of the individuals, and Bayesian initial priors were required on some of the parameters. These 
concerns were what prompted us to adopt a population approach, mixed effects modeling, which does not require fitting each individual's data separately. The population model, including data from all groups of individuals, was implemented in NONMEM. We chose to assume a population value of $0.03 \mathrm{~min}^{-1}$ for the insulin action parameter, $p_{2}$, as suggested previously (5). When an estimable fixed effect was added for $p_{2}$, not only was it estimated with poor precision, it also amplified the $\mathrm{CV}$ of the $\mathrm{S}_{1}$ estimate from $17.3 \%$ to $146 \%$, due to a strong inverse correlation between the two parameters. Furthermore, it did not improve the model fit, and was therefore not estimated in the final model. The parameter estimates for the final population model are given with their precisions in Table 2.

Plots of population predicted values versus observed concentrations showed considerable scatter around the line of unity (Fig. 2, top row). Specifically, the population predicted values do not capture the elevated concentrations of glucose, insulin and C-peptide observed in some individuals. These population predictions, however, are equivalent to having a single, "typical" prediction plotted against all the individual data and therefore reflect the kinetics of the entire population rather than any given individual. The identity line bisects the data, as expected, signifying a lack of any systematic error. To completely account for individual variation in the kinetic models, model parameters are individualized using empirical Bayes estimation, in a step that is subsequent to the estimation of the population mean parameters. When these empirical post hoc Bayes estimates obtained from the population approach were used to determine individually predicted values, these points collapsed to the line of unity, showing no systematic deviations from the observed data (Figure 2, bottom row).

Individual empirical Bayes parameter estimates were calculated and analyzed to determine their ability to detect differences between subjects with varying degrees of glucose tolerance. Normal subjects were found to have the highest median values for SI, as well as the most variability (Fig. 3). Mean estimates and standard errors for $\mathrm{S}_{\mathrm{I}}\left(10^{4}\right.$ $\left.\mathrm{min}^{-1} \cdot \mathrm{MU}^{-1} \cdot \mathrm{ml}\right)$ in the different groups were: Group1 (17.7 \pm 1.8$)$, Group $2(9.95 \pm 2.01)$, Group 3 (5.67 \pm 1.02$)$, Group 4 (3.82 \pm 1.12$)$ and Group 5 (9.39 \pm 3.82$)$. The presence of three outliers in the diabetic group (Group 5) suggests the model may tend to overestimate $S_{1}$ in extreme disease cases, inflating the group mean value. For instance, 
the subject with the highest estimate of $\mathrm{S}_{1}, 62.2 \times 10^{4} \mathrm{~min}^{-1} \cdot \mu \mathrm{U}^{-1} \cdot \mathrm{ml}$, also had both the highest fasting and 2-hour glucose concentrations. Exclusion of the three outliers in Group 5 resulted in a mean estimate in better agreement with expectations (3.41 \pm $0.64)$.

Individual insulin secretion profiles were also reconstructed from empirical Bayes estimates (Fig 4). As expected, diabetic subjects (Group 5) displayed a blunted and delayed insulin response to the OGTT, whereas subjects with elevated 2-hour glucose concentrations showed an augmented insulin response characteristic of subjects with impaired glucose tolerance.

\section{DISCUSSION}

Type 2 diabetes is characterized by either fasting plasma glucose of $\geq 126 \mathrm{mg} / \mathrm{dl}$, or by a 2-h plasma glucose of $\geq 200 \mathrm{mg} / \mathrm{dl}$ after a 75 -gram oral glucose load (OGTT), or both (21). The increase in hyperglycemia with age is almost entirely due to increases in 2-h glucose levels after an OGTT, with very little change in fasting glucose levels (22), and the prevalence of diabetic 2-h glucose levels in subjects over 70 years of age with a normal fasting glucose is $9 \%$. Normal glucose homeostasis depends on there being adequate insulin secretion and sensitivity. Study participants with both fasting and 2-h plasma glucose in the diabetic range had abnormalities in both insulin secretion and insulin sensitivity (worsening HOMA-IR and worsening insulinogenic indices), whereas subjects with normal fasting glucose levels but impaired and diabetic 2-h plasma glucose levels had, as their primary abnormality, deficient insulin secretion in the postprandial period-as evidenced by the worsening insulinogenic indices only.

In clinical practice, as well as in large scale diabetes outcomes trials, where high numbers of individuals have to be screened and follow-up for many months or even years, it is practically problematic and not cost effective to perform an OGTT on a population scale. Pragmatic circumstances around the conduct of trials may also be considerable limiting factors, diminishing adherence and potentially placing participant retention at risk. The fact that there appears to be a remarkable change in the conventional paradigm in randomized trials of individuals with diabetes, aiming at 
investigating interventions and pharmacological agents at gradually earlier stages of the disease process, would increasingly result in large numbers of people at the "prediabetic" state of glucose intolerance in need for thorough screening.

Reliable tools and population-based investigative techniques for qualitative classification of the prevailing patterns characterizing fasting and/or postprandial hyperglycemia, applicable to large numbers of people, are much needed given the diabetes and obesity epidemic, especially in developing countries. Such an approach, could aid in the early identification of those populations at risk of developing impaired glucose tolerance and eventually type 2 diabetes, based on their aberrant 2-h responses even in the presence of fasting euglycemia.

Although the technique of population mixed effects modeling has enjoyed a rich history in the fields of pharmacokinetic and pharmacodynamic data analysis, its application to metabolic studies, with exceptions $(10,23)$, is fairly limited. One possible explanation is that population analysis is naturally suited for data-poor experiments performed in a large number of subjects, whereas the IVGTT, as interpreted by the minimal model, is a data-rich experiment often performed in a small subset of individuals. Epidemiological studies like the Insulin Resistance Atherosclerosis Study (IRAS) (24) and the FinlandUnited States Investigation of NIDDM Genetics (FUSION) Study (25) are two exceptions in that they include IVGTT's with reduced sampling on a large number of study subjects. In addition to the IVGTT, the simpler OGTT is often performed on a separate day to assess glucose tolerance. It would therefore be economical in future studies to estimate indexes of insulin resistance and $\beta$-cell function from only a single OGTT. Achieving this goal requires not only appropriate structural models, but also novel means of data analysis. This work is aimed at the latter, demonstrating the feasibility of a population mixed effects modeling approach to analyze the sparse data of the OGTT in a large population with varying levels of glucose tolerance.

Population analysis, as implemented in NONMEM, provides estimates of population parameter means, variances and covariances in addition to empirical Bayes estimates of individual parameters. Other techniques, such as the standard two-stage (STS) and 
iterative two-stage (ITS) methods require individual estimates before population information can be inferred. In the model-building phase, we attempted this type of approach, but found individual model parameters were not estimable in over one-third of the subjects. However, this initial effort was helpful in establishing the model structure and suggesting initial parameter estimates. Furthermore, population parameter estimates were comparable to individual estimates, suggesting agreement between the two methods.

The mixed effects population approach has been applied to data obtained from glucose tolerance tests on at least one other occasion. De Gaetano et al. (10) estimated insulin sensitivity from IVGTT data in 20 normal subjects and found improved precision of the population mean and covariance estimates compared to the standard two-stage method. However, individual parameter estimates were not obtained, suggesting the population approach is only appropriate for comparing parameters in different populations. In the current study, we made use of the POSTHOC option in NONMEN to obtain empirical Bayes estimates of individual model parameters. This method, however, does not enable us to obtain precisions on the individual parameters. One possible problem with using a population approach to obtain individual estimates with sparse data is that these estimates may be regressed to the mean, concealing between-subject variability. Since our database included subjects with a wide range of glucose tolerances, we were able to show these posterior estimates do reflect underlying changes in glucose-insulin regulation, suggesting regression to the mean is negligible for this application. We do not, however, suggest that these estimates necessarily predict the five clinical states. To provide further validation for these methods, individual estimates will have to be further compared to those obtained from more established methods such as the IVGTT or the clamp.

We chose the oral glucose minimal model and the combined model to describe glucose and insulin kinetics during the OGTT. The population approach, however, can be applied to many different models of kinetic data. For example, we could have used spline functions to describe secretion rates, or used alternative models for glucose absorption (5) and insulin secretion (27). This work is primarily intended to serve as a "proof-of-concept" study, so the choice of model is not critical. Since not all model 
parameters were uniquely identifiable, we set parameters $\mathrm{V}$ and $\mathrm{S}_{\mathrm{G}}$ to be the same values in all subjects. Since there is disagreement whether changes in $\mathrm{S}_{G}$ are due to changes in glucose tolerance or are just a minimal model artifact (27), we assumed all subjects had the same parameter value. Likewise, all subjects were assumed to have the same value for the insulin action parameter, $p_{2}$. It is possible that some individuals' true parameters deviate from these population means. The extent to which these assumptions result in error of the $S_{I}$ estimates remains to be determined.

The population estimate of $S_{1}$ obtained in this work, $6.51 \times 10^{-4} \mathrm{~min}^{-1} \cdot \mu \mathrm{U}^{-1} \cdot \mathrm{ml}$, is in accordance with previous studies. The rather wide between-subject variability of insulin sensitivity, $113 \%$, is plausible, considering even groups of normal subjects show a wide range of variability. The inclusion of subjects with glucose intolerance would only amplify this variability. Likewise, the estimates obtained for $\mathrm{F}$ and $k$ / were both reasonable with considerable inter-subject variability, $\sim 45 \%$ for both.

One distinct advantage of population analysis not exploited in this study is the ability to incorporate demographic covariates into the mathematical model. In a study rich with anthropometric data, it might be of interest to determine how model parameters are affected by factors such as abdominal fat, age, weight, or genetic profile.

We believe that such modeling approaches may be critically important in determining rates of progression to diabetes, as well as the impact of therapies aiming at potentially delaying onset or preventing diabetes, particularly in large cohorts of individuals with IGT. The current trend in interventional randomized trials towards recruiting IGT populations, especially those at high risk for or with existing cardiovascular disease, reflects growing appreciation of the pathophysiology of the "diabetes continuum" and may well be the most suitable candidate population to exploit this model further. Combined with validated robust outcomes models in diabetes, such as the one based on the United Kingdom Diabetes Prospective Study (UKPDS), it may be of significant added value for Regulatory Authorities and clinical decision making bodies which shape clinical practice. 
In conclusion, we introduce the technique of nonlinear mixed effects population modeling as applied to data from the OGTT, and show its ability to estimate both population and individual parameter values. Furthermore, we were able to show this method was robust enough to distinguish individuals with varying degrees of glucose tolerances. This approach might prove a valuable tool in exploring large epidemiological datasets and should be validated further in large scale trial data incorporating multiple parameters.

\section{ACKNOWLEDGEMENTS}

We thank Ms Amalia Matziou for diligent technical assistance; Ms Rebecca Kostatou and Katerina Nestoridou, for excellent co-ordination of the study. This work was supported by an unrestricted educational grant to MJT from the Hellenic Biotechnology Association. 


\section{REFERENCES}

1. DeFronzo RA, Tobin JD, and Andres R. Glucose clamp technique: a method for quantifying insulin secretion and resistance. Am J Physio/237: E214-E223, 1979

2. Toffolo G, De Grandi F, and Cobelli C. Estimation of beta-cell sensitivity from intravenous glucose tolerance test C-peptide data. Knowledge of the kinetics avoids errors in modeling the secretion. Diabetes 44: 845-854, 1995

3. Bergman RN, Ider YZ, Bowden CR, and Cobelli C. Quantitative estimation of insulin sensitivity. Am J Physio/236: E667-677, 1979

4. Breda E, Cavaghan MK, Toffolo G, Polonsky KS, and Cobelli C. Oral glucose tolerance test minimal model indexes of beta-cell function and insulin sensitivity. Diabetes 50: 150-158, 2001

5. Dalla Man C, Caumo A, and Cobelli $C$. The oral glucose minimal model: estimation of insulin sensitivity from a meal test. IEEE Trans Biomed Eng 49: 419-429, 2002

6. Agbaje OF, Luzio SD, Albarrak Al, Lunn DJ, Owens DR, and Hovorka R. Bayesian hierarchical approach to estimate insulin sensitivity by minimal model. Clin Sci (Lond) 105: 551-560, 2003

7. Vicini $P$, and Cobelli $C$. The iterative two-stage population approach to IVGTT minimal modeling: improved precision with reduced sampling. Intravenous glucose tolerance test. Am J Physiol Endocrinol Metab 280: E179-E186, 2001

8. Whiting B, Kelman AW, and Grevel J. Population pharmacokinetics. Theory and clinical application. Clin Pharmacokinet 11: 387-401, 1986

9. Beal SL, and Sheiner LB. Estimating population kinetics. Crit Rev Biomed Eng 8: 195-222, 1982

10. De Gaetano A, Mingrone G, and Castageneto M. NONMEM improves group parameter estimation for the minimal model of glucose kinetics. Am J Physiol 271: E932-E937, 1996

11. American Diabetes Association Clinical Practice recommendations. Diagnosis and Classification of Diabetes Mellitus Diabetes Care 27: S5-S10, 2004 
12. Bruce, D.G., Chisholm, D.J., Storlien, L.H., and Kraegen, E.W. Physiological importance of deficiency in early prandial insulin secretion in non-insulindependent diabetes. Diabetes 37: 736-744, 1988

13. Matthews, D.R., Hosker, J.P., Rudenski, A.S., Naylor, B.A., Treacher, D.F., and Turner, R.C. Homeostasis model assessment: insulin resistance and beta-cell function from fasting plasma glucose and insulin concentrations in man. Diabetologia 28: 412-419, 1985

14. Theodorakis MJ, Carlson O, Muller DC, and Egan JM. Elevated plasma glucosedependent insulinotropic polypeptide (GIP) associates with hyperinsulinemia in impaired glucose tolerance. Diabetes Care 27: 1692-1698, 2004

15. Kjems LL, Christiansen E, Volund A, Bergman RN, and Madsbad S. Validation of methods for measurement of insulin secretion in humans in vivo. Diabetes 49: 580-588, 2000

16. Watanabe RM, Steil GM, and Bergman RN. Critical evaluation of the combined model approach for estimation of prehepatic insulin secretion. Am J Physio/274: E172-183, 1998

17. Van Cauter E, Mestrez F, Sturis J, and Polonsky KS. Estimation of insulin secretion rates from C-peptide levels. Comparison of individual and standard kinetic parameters for C-peptide clearance. Diabetes 41: 368-377, 1992

18. Davidian M, and Giltinan D. Nonlinear Models for Repeated Measurement Data. New York: Chapman and Hall, 1995.

19. Barrett PH, Bell BM, Cobelli C, Golde H, Schumitzky A, Vicini P, and Foster DM. SAAM II: Simulation, Analysis, and Modeling Software for tracer and pharmacokinetic studies. Metabolism 47: 484-492, 1998

20. Beal SL, and Sheiner LB. NONMEM User Guide. San Francisco, CA: NONMEM Project Group, Univ. of California San Francisco, 1992.

21. Unwin N, Shaw J, Zimmet $P$, and Alberti KGMM. Impaired glucose tolerance and impaired fasting glycaemia: the current status on definition and intervention. Diabet Med 19: 708-723, 2002 
22. Elahi D, Muller DC, McAloon-Dyke M, Tobin JD, and Andres R. The effect of age on insulin response and glucose utilization during four hyperglycemic plateaus. Exp Geronto/28: 393-409, 1993

23. Rostami-Hodjegan A, Peacey SR, George E, Heller SR, and Tucker GT. Population-based modeling to demonstrate extrapancreatic effects of tolbutamide. Am J Physio/274: E758-E771, 1998

24. Wagenknecht LE, Mayer EJ, Rewers M, Haffner S, Selby J, Borok GM, Henkin L, Howard G, Savage PJ, Saad MF, Bergman RN, and Hamman R. The insulin resistance atherosclerosis study (IRAS) objectives, design, and recruitment results. Ann Epidemio/5: 464-472, 1995

25. Valle T, Tuomilehto J, Bergman RN, Ghosh S, Hauser ER, Eriksson J, Nylund SJ, Kohtamaki K, Toivanen L, Vidgren G, Tuomilehto-Wolf E, Ehnholm C, Blaschak J, Langefeld CD, Watanabe RM, Magnuson V, Ally DS, Hagopian WA, Ross E, Buchanan TA, Collins F, and Boehnke M. Mapping genes for NIDDM. Design of the Finland-United States Investigation of NIDDM Genetics (FUSION) Study. Diabetes Care 21: 949-958, 1998

26. Schirra J, Katschinski M, Weidmann C, Schafer T, Wank U, Arnold R and Goke B. Gastric emptying and release of incretin hormones after glucose ingestion in humans. J Clin Invest. 1996 Jan 1; 97(1): 92-103.

27. Toffolo G, Breda E, Cavaghan MK, Ehrmann DA, Polonsky KS, and Cobelli C. Quantitative indexes of beta-cell function during graded up\&down glucose infusion from C-peptide minimal models. Am J Physiol Endocrinol Metab 280: E2-E10, 2001

28. Finegood DT, and Tzur D. Reduced glucose effectiveness associated with reduced insulin release: an artifact of the minimal-model method. Am J Physiol 271: E485-E495, 1996

29. Theodorakis MJ, Carlson O, Michopoulos S, Doyle ME, Juhaszova M, Petraki K and Egan JM. Human duodenal enteroendocrine cells: source of both incretin peptides, GLP-1 and GIP. Am J Physiol Endocrinol Metab 290:E550-559, 2006 


\section{Supplement}

\section{Model structure}

The rate of change of glucose concentration with time is given by,

$$
\begin{array}{ll}
\frac{d G(t)}{d t}=-\left[S_{G}+X(t)\right] \cdot G(t)+S_{G} \cdot G_{b}+\frac{R_{a}(t)}{V} & G(0)=G_{b} \\
\frac{d X(t)}{d t}=-p_{2} \cdot\left\{X(t)-S_{I} \cdot\left[I(t)-I_{b}\right]\right\} & X(0)=0
\end{array}
$$

where $\mathrm{G}$ is plasma glucose concentration $\left(\mathrm{mg} \cdot \mathrm{dl}^{-1}\right)$, I is plasma insulin concentration $\left(\mu \mathrm{U} \cdot \mathrm{ml}^{-1}\right), G_{b}$ and $I_{b}$ are their basal values, $X$ is insulin action $\left(\mathrm{min}^{-1}\right)$ and $V$ is the volume of distribution of glucose $\left(\mathrm{dl} \cdot \mathrm{kg}^{-1}\right) . \mathrm{S}_{\mathrm{G}}\left(\mathrm{min}^{-1}\right)$ represents glucose effectiveness, reflecting the ability of glucose per se to stimulate glucose disposal and inhibit glucose production. $S_{I}\left(\min ^{-1} \cdot \mu \mathrm{U}^{-1} \cdot \mathrm{ml}\right)$ measures insulin sensitivity, or the ability of insulin to enhance the glucose stimulation of glucose disposal and glucose inhibition of glucose production. The minimal model parameter $p_{2}\left(\mathrm{~min}^{-1}\right)$ is the insulin action parameter. $\mathrm{R}_{\mathrm{a}}$, the posthepatic appearance of glucose, was described by a piecewise linear model with break points at $0,10,30,60,90$, and 120 minutes. Beyond the 120 sampling period, $R_{a}$ was assumed to obey mono-exponential decay with a decay rate of $\alpha=0.017 \mathrm{~min}^{-1}(5)$. The resulting expression for glucose appearance is thus,

$$
R_{a}(t)= \begin{cases}k_{i-1}+\frac{k_{i}-k_{i-1}}{t_{i}-t_{i-1}} \cdot\left(t-t_{i-1}\right), & \text { for } t_{i-1} \leq t \leq t_{i}, i=1 \ldots 5 \\ k_{5} \cdot \exp \left(-\alpha \cdot\left(t-t_{5}\right)\right) & \text { for } t>120\end{cases}
$$

The number of estimated glucose appearance rate parameters $k$ 's, was reduced by one by assuming $86 \%$ of the ingested glucose dose is absorbed into the circulation (5). A further reduction in the number of parameters was possible by noting that $k_{0}=0$ since $R_{a}(0)=0$. Since $S_{G}$ is non-uniquely identifiable and $V$ is non-identifiable, population values of $0.014 \mathrm{~min}^{-1}$ and $1.7 \mathrm{dl} / \mathrm{kg}$ were assumed as suggested previously (5). The resulting vector of estimable parameters of the oral glucose minimal model for each subject is therefore $\beta_{1}=\left\{p_{2}, S_{1}, k_{2}, k_{3}, k_{4}, k_{5}\right\}$.

C-peptide kinetics were described by the familiar two compartment model, 


$$
\begin{array}{ll}
\frac{d C P_{1}(t)}{d t}=-\left(k_{01}+k_{21}\right) \cdot C P_{1}(t)+k_{12} C P_{2}(t)+S R(t) & C P_{1}(0)=0 \\
\frac{d C P_{2}(t)}{d t}=k_{21} C P_{1}(t)-k_{12} C P_{2}(t) & C P_{2}(0)=0
\end{array}
$$

where $\mathrm{CP}_{1}\left(\mathrm{pmol} \cdot \mathrm{I}^{-1}\right)$ is the $\mathrm{C}$-peptide concentration above basal in the accessible compartment, $\mathrm{CP}_{2}$ is the equivalent concentration in the peripheral compartment, equal to the mass of $\mathrm{C}$-peptide in the peripheral compartment divided by the volume of distribution of the accessible compartment. The kinetic parameters $k_{12}$ and $k_{21}\left(\mathrm{~min}^{-1}\right)$ describe the rates at which C-peptide is transferred between the two compartments. Irreversible loss from the accessible compartment is represented by the rate parameter $k_{01}\left(\mathrm{~min}^{-1}\right)$. Kinetic parameters, $k_{i j}$ s, were calculated using prior knowledge of population C-peptide kinetics as outlined previously (17). SR(t) $\left(\mathrm{pmol}^{-1} \cdot \mathrm{min}^{-1}\right)$, the secretion rate of C-peptide above basal entering the accessible compartment normalized to the volume of distribution of the accessible compartment, was described by a piecewise linear function similar to $R_{a}$ above, but with rate parameters $j i$ 's. Also, insulin secretion was modeled only for the 120 minute duration of the OGTT. Once again, initial conditions dictate $j_{0}=0$. Thus, the vector of estimable parameters from the C-peptide model is given by $\beta_{2}=\left\{j_{1}, j_{2}, j_{3}, j_{4}, j_{5}\right\}$. Insulin is assumed to be secreted by the $\beta$-cells into a central compartment from which it is eliminated by a first-order process. The rate of change of insulin kinetics was described by a one compartment model,

$$
\frac{d I(t)}{d t}=-k_{I} I(t)+F \cdot S R(t) \quad I(0)=0
$$

where I $\left(\mu \mathrm{U} \cdot \mathrm{ml}^{-1}\right)$ is insulin concentration above basal and $k_{/}\left(\mathrm{min}^{-1}\right)$ is the irreversible loss from the single insulin compartment. $F$ is the fraction of insulin lost to hepatic extraction normalized by the ratio of the $\mathrm{C}$-peptide and insulin volumes of distribution (16). Since insulin is measured in $\mu \mathrm{U} \cdot \mathrm{ml}^{-1}$ and C-peptide is measured in pmol. $\mathrm{l}^{-1}$, this term must also be normalized by the ratio of units. Therefore, the term $\mathrm{F} \cdot \mathrm{SR}(\mathrm{t})$ is the secretion rate of pancreatic insulin reaching the systemic circulation. The modelpredicted time course of insulin concentration, $I(t)$, is used as the input to the model of glucose kinetics in Equation 2 above. Besides the rate parameters, jis, the vector of parameters specific to the insulin secretion model is defined as $\beta_{3}=\{k, F\}$ making the 
final vector of estimable parameters from both the oral glucose minimal model and the combined model, $\beta=\left[\beta_{1}, \beta_{2}, \beta_{3}\right]=\left\{p_{2}, S_{1}, k_{2}, k_{3}, k_{4}, k_{5}, j_{1}, j_{2}, j_{3}, j_{4}, j_{5}, \mathrm{k}_{1}, \mathrm{~F}\right\}$.

The general form of a nonlinear mixed effects model for multiple responses (i.e. glucose, insulin, and C-peptide) is given by (18),

$$
y_{i j}=f_{k}\left(\mathbf{x}_{i j}, \beta_{i}\right)+\varepsilon_{i j}
$$

where $y_{i j}$ denotes the $j$ th response from the $i$ th individual. The function $f$ is common to all individuals and represents the value predicted from the structural models described in the previous section. The index $k=1,2,3$ refers to the fact there are three separate models for glucose, C-peptide and insulin concentrations. The vector $\mathbf{x}_{\mathrm{ij}}$ incorporates fixed values of covariates for each individual, including age, weight, height, and basal concentrations of glucose, insulin and C-peptide. For example, the function $f_{1}$ relates to the model prediction of $\mathrm{G}(\mathrm{t})$ determined by the solution to Equation 1 given above. In this case, the $y_{i j}$ 's refer to the directly observable, noisy measurement of glucose concentrations in plasma. The vector $\mathbf{x}_{\mathbf{i j}}$ includes model inputs such as the basal levels of glucose and insulin, $G_{b}$ and $I_{b}$. For the $C$-peptide model, $f_{2}$ is the solution of Equation 4 since $\mathrm{CP}_{1}(\mathrm{t})$ is the model prediction of the observed concentrations of $\mathrm{C}$-peptide in the plasma. The vector $\mathbf{x}_{\mathrm{ij}}$ now holds subject-specific values of height, weight, age and gender, which are used to calculate the kinetic parameters, $k_{i j}$ 's, in Equations 4 and 5. The function $f_{3}$ refers to the solution of Equation 6 , which the model prediction for the time course of insulin, $I(t)$. The $y_{i j}$ 's are now the observed insulin concentrations and $x_{i j}$ is the basal insulin concentration, $\mathrm{I}_{\mathrm{b}}$. The residual unexplained intra-individual error is described by the random variable $\varepsilon_{\mathrm{ij}}$, symmetrically distributed around 0 with variance $\sigma^{2}$. The parameter vector, $\beta_{i}$, is comprised of fixed and random effects components and can be expressed by the following,

$$
\beta_{i}=\beta_{i}\left(\beta_{p o p}, \eta_{i}\right)
$$

where $\beta_{p o p}$ represents the vector of fixed population mean parameters and $\eta_{i}$ reflects the random deviation of an individual's parameters from the population means. For the functions $f_{1}, f_{2}$, and $f_{3}$, the corresponding parameter vectors are $\beta=\left\{p_{2}, S_{1}, k_{2}, k_{3}, k_{4}, k_{5}\right\}$, $\boldsymbol{\beta}=\left\{j_{1}, j_{2}, j_{3}, j_{4}, j_{5}\right\}$ and $\boldsymbol{\beta}_{3}=\left\{k_{1}, F\right\}$, corresponding to the models of glucose, C-peptide and insulin, respectively. Between-subject variability was explicitly modeled for each 
parameter. For example, the $n$th element of the ith individual's parameter set can be assumed to be one realization from an underlying population distribution which is lognormal so that,

$$
\begin{gathered}
\beta_{i, n}=\beta_{p o p, n} \cdot \exp \left(\eta_{i}\right), \quad \eta \sim \mathrm{N}(0, \omega) \\
\text { where } \beta_{i, n} \sim \operatorname{LN}\left(\beta_{p o p, n}, \omega\right)
\end{gathered}
$$

Alternatively, some parameters were modeled with an additive random effect term implying that each individual parameter value arises from an underlying normal distribution so that,

$$
\begin{gathered}
\beta_{i, n}=\beta_{p o p, n}+\eta_{i}, \quad \eta \sim \mathrm{N}(0, \omega) \\
\text { where } \beta_{i, n} \sim \mathrm{N}\left(\beta_{p o p, n}, \omega\right)
\end{gathered}
$$

The variances and covariances among the components of $\eta$ are contained in a joint covariance matrix, $\Omega$, with diagonal elements $\left(\omega_{1}{ }^{2}, \omega_{2}{ }^{2}, \ldots, \omega_{n}{ }^{2}\right)$, allowing for correlations among the parameter values. 


\section{FIGURE LEGENDS}

\section{FIGURE 1}

2-h profiles of plasma glucose (A), insulin (B), and C-peptide (C), intact (N-terminal) responses during the oral glucose tolerance test in all five groups of subjects. Glucose $(75 \mathrm{~g})$ was administered at time 0 . Data is shown as means \pm SE.

\section{FIGURE 2}

Population and individual predicted versus observed concentrations of glucose insulin and C-peptide after fitting of final kinetic model using the population approach. The top row shows the single "typical" population predicted value vs. the observed measurements. The bottom row shows the individualized predictions based on empirical Bayes estimates vs. the observed measurements.

\section{FIGURE 3}

Boxplot of estimates obtained from the population model for the five different groups with varying levels of fasting glucose and glucose tolerance. The bottom and top lines of the box represent the $25^{\text {th }}$ and $75^{\text {th }}$ percentiles, respectively. The horizontal line inside the box represents the median of the data. Whiskers project 1.5 times the length of the box, with outliers represented with $(+)$. Median values $\left(\mathrm{min}^{-1} \cdot \mu \mathrm{U}-1 \cdot \mathrm{ml}\right)$ are: Group 1 $=15.0$, Group $2=8.28$, Group $3=4.34$, Group $4=2.73$ and Group $5=3.67$.

\section{FIGURE 4}

Mean insulin secretion profiles for different groups obtained from the final population kinetic model. 
Population analysis of the oral glucose tolerance test

\begin{tabular}{|c|c|c|c|c|c|}
\hline \multirow{3}{*}{ VARIABLE } & \multicolumn{3}{|c|}{ GLUCOSE TOLERANCE } & \multicolumn{2}{|c|}{$\begin{array}{l}\text { 2-HOUR POST-CHALLENGE } \\
\text { PLASMA GLUCOSE }\end{array}$} \\
\hline & $\begin{array}{l}\text { NORMAL } \\
\text { (N=41) }\end{array}$ & $\begin{array}{l}\text { MPAIRED } \\
\text { (N=20) }\end{array}$ & $\begin{array}{l}\text { TYPE } 2 \text { DIABETES } \\
\qquad(\mathrm{N}=17)\end{array}$ & $\begin{array}{l}\text { MPAIRED } \\
\text { (N=19) }\end{array}$ & $\begin{array}{l}\text { DIABETIC } \\
\text { (N=9) }\end{array}$ \\
\hline & Group 1 & Group 3 & Group 5 & Group 2 & Group 4 \\
\hline Age (years) & $71 \pm 2$ & $73 \pm 2$ & $71 \pm 2$ & $77 \pm 2$ & $79 \pm 3$ \\
\hline Range & $39-91$ & $58-79$ & $54-87$ & $62-91$ & $66-91$ \\
\hline Gender (F/M) & $20 / 21$ & $10 / 10$ & $3 / 14$ & $10 / 9$ & $5 / 4$ \\
\hline Body Mass Index $\left(\mathrm{kg} / \mathrm{m}^{2}\right)$ & $27 \pm 1$ & $29 \pm 1$ & $30 \pm 2$ & $28 \pm 1$ & $27 \pm 2$ \\
\hline Range & $19-39$ & $21-38$ & $23-40$ & $22-38$ & $20-36$ \\
\hline Hemoglobin $\mathrm{A}_{1 \mathrm{c}}\left(\mathrm{HbA}_{1 \mathrm{c}} \text { \% }\right)^{\prime}$ & $5.31 \pm 0.08$ & $5.88 \pm 0.17^{ \pm}$ & $6.97 \pm 0.22^{\circ}$ & $5.44 \pm 0.11$ & $5.92 \pm 0.20^{5.7}$ \\
\hline Fasting plasma glucose (mg/dl) & $9 \theta \pm 1$ & $108 \pm 1^{*}$ & $147 \pm 4^{201}$ & $90 \pm 2$ & $87 \pm 3^{*}$ \\
\hline Fasting plasma insulin ( $\mu \mathrm{U} / \mathrm{ml})$ & $7 \pm 1$ & $9 \pm 1$ & $11 \pm 1^{*}$ & $6 \pm 1$ & $8 \pm 2$ \\
\hline Fasting plasma glucagon (pmol/) & $68 \pm 4$ & $73 \pm 16$ & $107 \pm 12^{*}+$ & $68 \pm 6$ & $73 \pm 6$ \\
\hline HOMA-IR & $1.46 \pm 0.17$ & $2.34 \pm 0.33$ & $3.80 \pm 0.43^{x .06}$ & $1.73 \pm 0.22$ & $1.55 \pm 0.31$ \\
\hline Insulinogenic Index & $0.87 \pm 0.08$ & $0.47 \pm 0.12$ & $0.34 \pm 0.11^{*}$ & $0.63 \pm 0.11$ & $0.30 \pm 0.05^{x, t}$ \\
\hline Total Cholesterol (mg/dl) & $192 \pm 7$ & $191 \pm 9$ & $184 \pm 8$ & $214 \pm 10$ & $198 \pm 10$ \\
\hline HDL Cholesterol (mg/dl) & $60 \pm 3$ & $48 \pm 3^{*}$ & $43 \pm 2^{t}$ & $55 \pm 4$ & $58 \pm 4$ \\
\hline LDL Cholesterol (mg/dl) & $116 \pm 8$ & $118 \pm 7$ & $100 \pm 7$ & $130 \pm 9$ & $118 \pm 8$ \\
\hline Triglycerides (mg/dl) & $83 \pm 6$ & $128 \pm 22$ & $204 \pm 35^{*}$ & $124 \pm 17$ & $111 \pm 15$ \\
\hline
\end{tabular}

T $\mathrm{HbA}_{1 \mathrm{c}}$ denotes glycosylated hemoglobin

* denotes statistically significant difference compared to subjects with normal glucose tolerance $\left({ }^{*} P<0.05 ;{ }^{\star *} P<0.001\right)$

"denotes statistically significant difference compared to subjects with impaired glucose tolerance $\left({ }^{*} P<0.05 ;{ }^{*} P<0.001\right)$

${ }^{\dagger}$ denotes statistically significant difference compared to subjects with impaired 2 hour glucose $\left({ }^{\dagger} P<0.05\right)$ 
FIGURE 1

\section{Glucose}
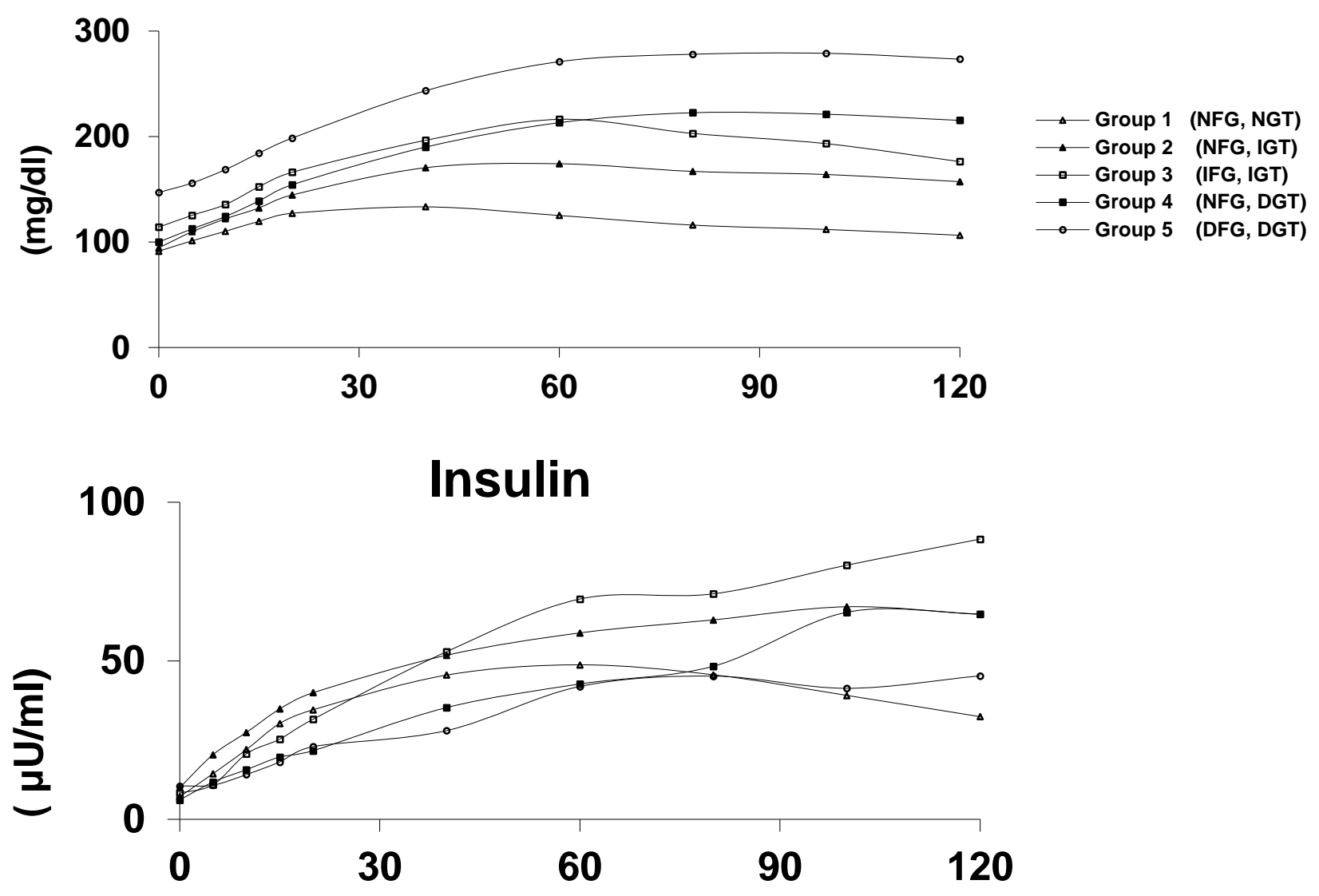

\section{C-peptide}

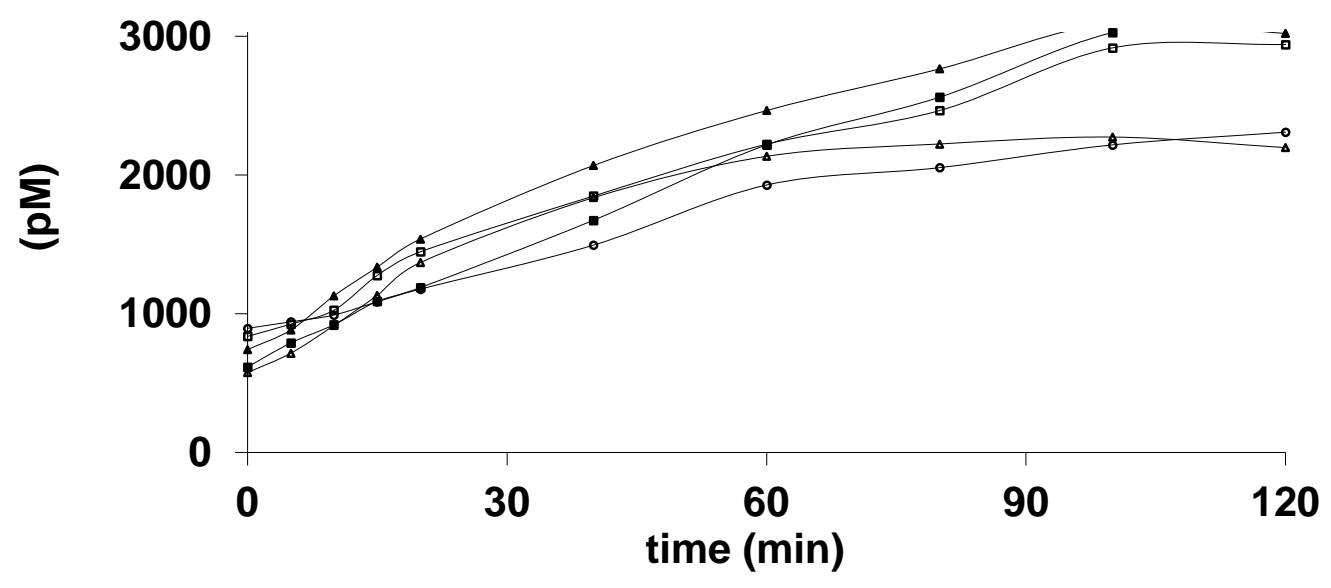


FIGURE 2
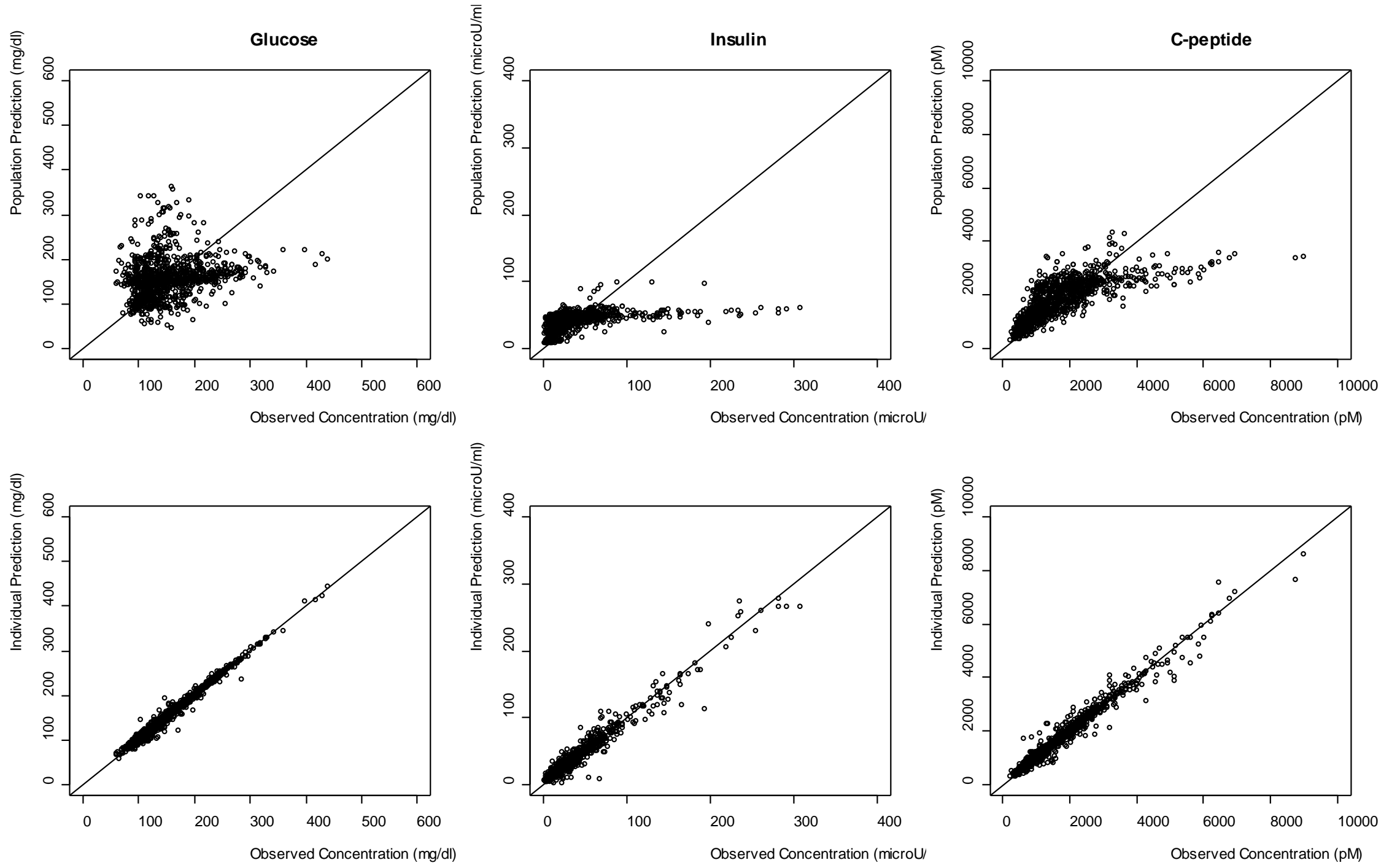
Table 2. Population model parameters describing glucose and insulin kinetics during the OGTT.

* Variability is given in \% for terms describing proportional error

\begin{tabular}{|c|c|c|c|c|c|c|}
\hline & \multicolumn{2}{|c|}{ Parameter } & \multicolumn{2}{|c|}{ Between-subject variability } & \multicolumn{2}{|c|}{ Residual Variability } \\
\hline & Estimate & Standard Error of Estimate & Estimate & Standard Error of Estimate & Estimate & Standard Error of Estimate \\
\hline $\begin{array}{l}\mathrm{S}_{\text {I }} \\
\quad\left(10^{-4} \cdot \mathrm{m}\right.\end{array}$ & 6.51 & 1.20 & $113 \%^{*}$ & $29.6 \%^{*}$ & & \\
\hline$k_{2}$ & 5.07 & 0.251 & 1.97 & 0.690 & & \\
\hline$k_{3}$ & 6.54 & 0.255 & 2.43 & 0.581 & & \\
\hline$k_{4}$ & 7.09 & 0.323 & 2.89 & 0.642 & & \\
\hline$k_{5}$ & 6.62 & 0.380 & 4.24 & 0.751 & & \\
\hline $\mathrm{F}$ & 0.522 & 0.291 & $47.2 \%{ }^{*}$ & $30.3 \% *$ & & \\
\hline$k^{\prime} \quad\left(\min ^{-1}\right)$ & 0.258 & 0.151 & $43.2 \%^{*}$ & $26.6 \%{ }^{*}$ & & \\
\hline${ }^{j 1} \quad(\mathrm{pmol} \cdot \mathrm{m}$ & 106 & 13.7 & 77.7 & 22.1 & & \\
\hline${ }^{j 2} \quad(\mathrm{pmol} \cdot \mathrm{m}$ & 91.3 & 6.45 & 51.0 & 9.79 & & \\
\hline $\begin{array}{l}J_{3} \\
\quad(\mathrm{pmol} \cdot \mathrm{m}\end{array}$ & 129 & 10.8 & 73.4 & 17.0 & & \\
\hline${ }^{j_{4}} \quad(\mathrm{pmol} \cdot \mathrm{m}$ & 133 & 13.6 & 89.4 & 21.4 & & \\
\hline${ }^{j_{5}} \quad(\mathrm{pmol} \cdot \mathrm{m}$ & 117 & 17.6 & 94.0 & 35.2 & & \\
\hline Proportiona & & & & & & \\
\hline Glucose & & & & & $6.9 \%^{*}$ & $1.21 \%^{*}$ \\
\hline Insulin & & & & & $26.5 \%^{*}$ & $6.39 \%{ }^{*}$ \\
\hline C-peptide & & & & & $13.6 \%{ }^{*}$ & $2.66 \%{ }^{*}$ \\
\hline
\end{tabular}


Population analysis of the oral glucose tolerance test

FIGURE 3

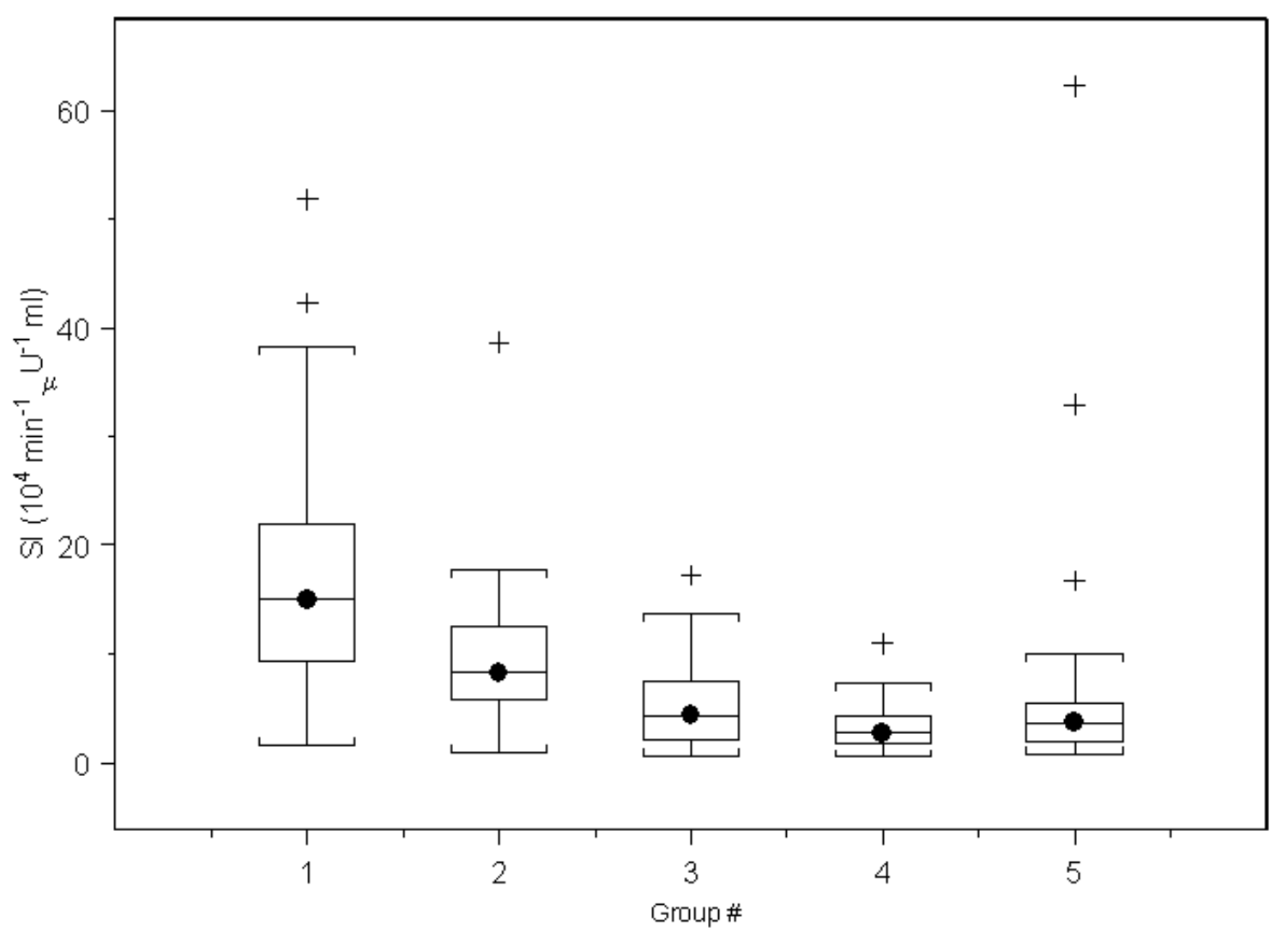




\section{FIGURE 4}

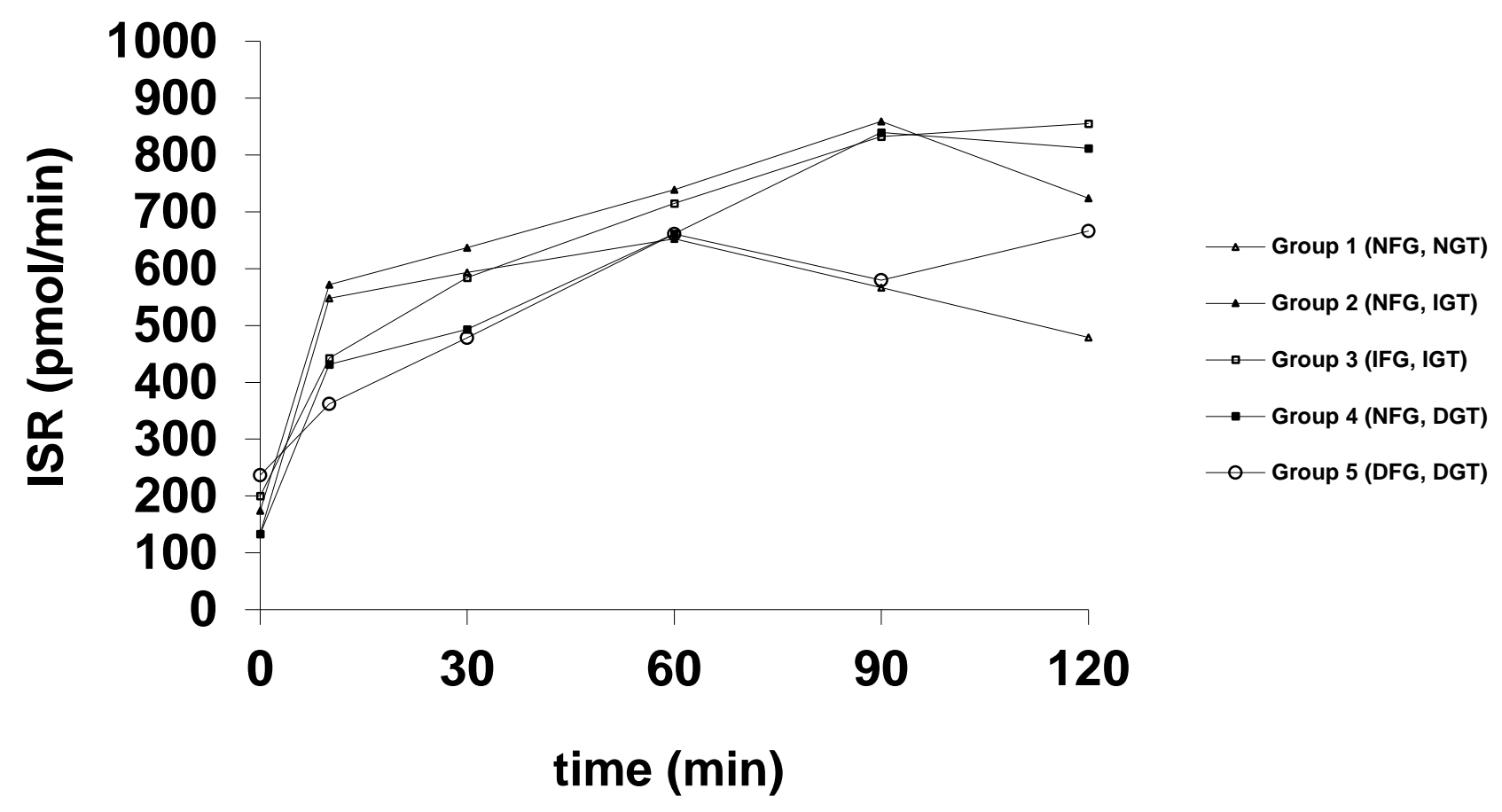

